\title{
GENETIC ANALYSIS OF POSSIBLY THE OLDEST GREYHOUND REMAINS WITHIN THE TERRITORY OF THE CZECH REPUBLIC AS PROOF OF A LOCAL ELITE PRESENCE AT CHOTĚBUZ-PODOBORA HILLFORT IN THE $8^{\text {TH }}-9^{\text {TH }}$ CENTURY AD
}

\author{
HANA SVOBODOVÁ \\ Department of Criminalistic Technique and Expertise, Police of the Czech Republic, Kounicova 24, 61132 Brno, the Czech \\ Republic; email: hancin@seznam.cz
}

MILAN BARTOŠ

Department of Molecular Biology and Pharmaceutical Biotechnology, Veterinary and Pharmaceutical University, Palackého trr. 1/3, 612 42, Brno, the Czech Republic; email: bartosm@VFU.cz

MIRIAM NÝVLTOVÁ FIŠÁKOVÁ

Institute of Archaeology, Czech Academy of Science, Čechyňská 19, 602 00, Brno, the Czech Republic; email: nyvltova@arub.cz

PAVEL KOUŘIL

Institute of Archaeology, Czech Academy of Science, Čechyňská 19, 602 00, Brno, the Czech Republic; email: kouril@arub.cz

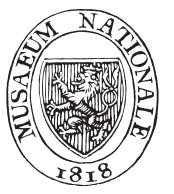

Svobodová, H., Bartoš, M., Nývltová Fišáková, M., Kouřil, P. (2015): Genetic analysis of possibly the oldest greyhound remains within the
territory of the Czech Republic as a proof of a local elite presence at Hotěbuz-Podobora hillfort in the $8^{\text {th }} 9^{\text {th }}$ century AD. - Acta Mus. Nat. Pragae, Ser. B Hist. Nat., 71(1-2): 17-24. Praha. ISSN 1804-6479.

Abstract. Very gracile dog bones dated to the $8^{\text {th }}-9^{\text {th }}$ century $\mathrm{AD}$ were found at the Chotěbuz-Podobora archaeological site in the Czech part of Silesia near the town of Český Těšín. Osteological analysis indicated that the bones were from a greyhound. To confirm this hypothesis, genetic analysis of greyhound-specific Single nucleotide polymorphisms (SNP) in the control region (CD, also D-loop) of the mitochondrial DNA (mtDNA) was performed, together with analysis of buccal swabs from living greyhounds from the Czech Republic. Our genotyping results strongly support the hypothesis that bones found were from a greyhound which supports the second hypothesis that an elite order inhabited Chotěbuz-Podobora hillfort in the Middle Ages.

greyhound; mtDNA; Control region; Great Moravia; Middle Ages

Received February 11, 2015

Issued October, 2015

\section{Introduction}

The Chotěbuz-Podobora hillfort lies $5 \mathrm{~km}$ northwest of the town of Český Těšín. The hillfort is situated on featureless plateaus, which were formed by the fluvial sediments of the main terrace of the Olše River and then covered by loess loams. The terrace hangs over the concave bank of the Olše River.

The hillfort was built by Lusatian Culture people $\left(10^{\text {th }}\right.$ to $5 / 4^{\text {th }}$ century BC). The Slavonic settlement is dated from the $8^{\text {th }}$ century to the first part of the $11^{\text {th }}$ century AD (Kouril 1994). The people who lived there controlled a site of strategic importance: the exit through the Moravian Gate, the main European connection between North and South (Kouril 1997, 2005). Other evidence of a local elite presence at the Chotěbuz-Podobora hillfort at that time is the finding of log house remains and paling structures on the acropolis as well as the finds of militaria (axes, spears, and arrow-heads) and riding equipment (spurs with hooks and discs, stirrups, bits, and buckles). The spurs in particular can be considered to be a significant emblem of the Slavonic mounted units, as a statutory symbol and feature of free men and warriors supporting the local leader. In addition to these militaria, an abundance of jewellery was also found (silver-plated earrings, rings and beads). The finding of two limb bones of a greyhound-like dog at the acropolis is also associated with the aforementioned features .

The bones of the greyhound-like dog were located together with other osteological material originating from the dense cultural layer found in the probe pit S-44 at the acropolis at a depth of 100-145 cm below the surface (Nývltová Fišáková 2007). This layer relates to the older phase of Slavonic settlement which is archaeologically dated to the second half of the $8^{\text {th }}$ century and $9^{\text {th }}$ century (Kouril 1994). The discovered skeletal remains of the greyhound-like dog were dated at $830 \pm 49 \mathrm{AD}$ cal. (AMS radiocarbon dating Poz-20508; calibration using IntCal13 dataset - Reimer et al. 2013). This age clearly correlates with the aforementioned archaeological assumptions. The test pit was located in the northernmost part of the acropolis, in the best-protected section of the whole fortification. The limb bones are long, slim, and flattened front-to-back (Text-fig. 1). The dog's height is calculated as $70 \mathrm{~cm}$ according to Harcourt's (1974) method (Nývltová Fišáková 2007, 2010). The ratio between 


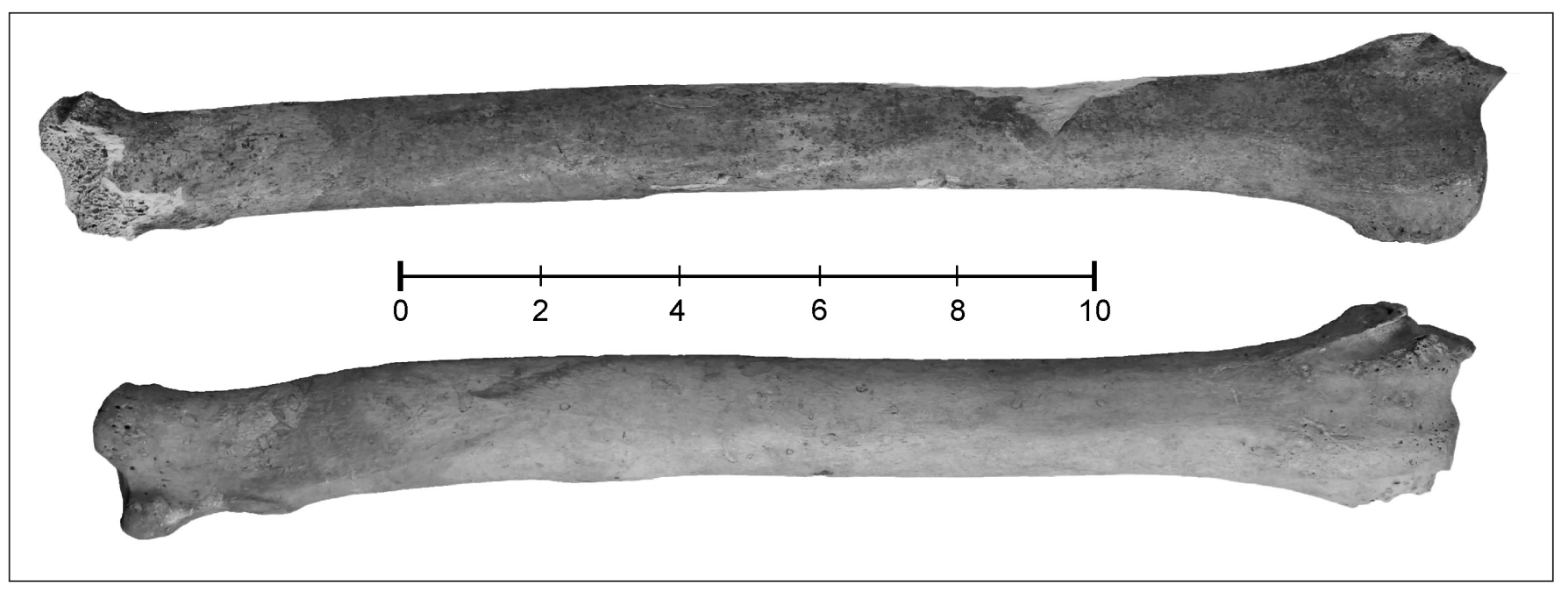

Text-fig. 1. Bottom view of the spoke bone of the excavated greyhound-like dog (above) and the spoke bone of a dog of the same size category (below). The gracility of the spoke bone of the greyhound-like dog is clearly visible. Photo by D. Nývlt.

the maximum length and the minimum width falls into the variation width of the ratio for greyhounds, mainly Polish greyhounds (Nývltová Fišáková 2007, 2010, fig. 8).

The greyhound has been considered an exceptional animal since the very earliest times (Sullivan 1999, Morris 2001, Branigan 2004). During the Middle Ages these dogs could only have been owned by nobles and rulers (Kholová 1987, Stuchlý and Císařovský 1992, Sullivan 1999, Branigan 2004, Císařovský 2008). The greyhound was one of the heraldic symbols of kings and nobles in both England and France (Sullivan 1999, Branigan 2004), as well as in Czech lands (Mysliveček 1993, Buben 2003). This is the oldest evidence of a greyhound in the Czech Republic. Until this discovery, the oldest finding was the mummified English greyhound from the castle in Ždánice (Nývltová Fišáková, 2007).

The main aim of this study was to analyze 17 excavated bones to test the hypothesis that these were the oldest greyhound remains within the Czech territory. We chose to analyse mitochondrial DNA (mtDNA) because of its special features: it is situated the outside nucleus in mitochondria, there are many copies while there are usually only two copies of each chromosome. Circular mtDNA is also more stable than linear nDNA, thus increasing the chance to obtain analyzable results even from degraded (ancient) DNA.

The first published complete mitochondrial DNA (mtDNA) genome of the domestic dog (Canis familiaris) contained $16,727 \mathrm{bp}$ (base pairs) with the control region (CR) spanning positions $15,458-16,727$ (1,270 bp) (Kim et al. 1998). While the dog mtDNA genome closely resembles the mtDNA genome of other mammals, the dog (and other related canids) mtDNA CR differs due to the presence of a 10 bp repeating unit (5'-GTACACGT(A/G)C-3') that begins at base 16,130 and varies in number and sequence both within and among individuals. The CR of dog mtDNA, like that of human mtDNA, has been the subject of a number of studies investigating variation among individuals (Vila et al. 1997, Kim et al. 1998, Webb and Allard 2009). Previous studies revealed more than 100 single nucleotide polymorphisms (SNPs) within the mtDNA CR of the domestic dog.

\section{Materials and methods}

The ancient material was found at Chotěbuz-Podobora site. These bones were osteologically very similar to greyhound-like dogs - long and generally slim in comparison with other dogs of a similar height (Text-fig. 1).

For comparison with recent domestic dog, we took a buccal swab from a greyhound whose ancestors were born in 1840 in Great Britain. We also took swabs from 15 recent dogs (greyhound-like dogs) from the dog exhibition in Brno 2010 as suitably representative samples for verification of our results. Sample 1 is Romanov z Palatinu Morava (male), sample 2 is Body z Murky (male) (both are Russian barsoi). Sample 3 is Limia Lykon (female, afghan greyhound). Sample 4 is Glenoak Junah (female, Saluki). Samples 5 and 6 are Lajjina and Pussy Bohemia Genao (both Sloughi). Sample 7 is Arabian Sloughi Djawida EJJI Fatira of Love ARAB BEAUTY. MAYSUN Bohemia Genao is sample 8, whose grand-father was a Moroccan Sloughi. Sample 9 Takellaut N8FA BADAKA is a Sloughi-Azawakh, whose ancestor was from the Sahara. Sample 10 is a Polish greyhound Baldwin-Xanthusia (male). Uncle Teddy (male) and Hellega (female) are whippets, samples 11 and 12 . Sample 13 is deerhound Greyrory's Draeura (female). Rendel (male) and Rosarka (female) are samples 14 and 15 are also barsois.

\section{Isolation of DNA from ancient bone}

Forensic DNA isolation methods were used which are a combination of the isolating methods described in the publications of Baron et al. (1996) and Yang et al. (1997). First we isolated DNA from the bone. The surfaces of both small bone fragments were mechanically cleaned with sandpaper and $96 \%$ ethanol was used to remove foreign substances, such as bacteria, fungi, or other foreign DNA. Usage of a more efficient bleaching liquid could be severely damaging for old bone. The next step of preparation for isolation of DNA was cutting the bone into thin slices with 
an oscillatory handsaw (Eickemeyer). The slices were crushed with a lever press and then ground with a mill (Retsch MM 200) for 3 minutes. The grindings were collected into agate bowls. The final volume of bone powder was $3.73 \mathrm{~g}$ and $3.54 \mathrm{~g}$ in $50 \mathrm{ml}$ tubes. The isolation process itself was done according to a protocol using phenol-chloroformisoamylalcohol. A total of $40 \mathrm{ml}$ of EDTA ( $\mathrm{pH} \mathrm{8.0)}$ was added to the bone powder and the solution was incubated for 24 hours at $4{ }^{\circ} \mathrm{C}$ (the process of decalcification) and then centrifuged at 4,000 rpm for 15 minutes. Forty $\mathrm{ml}$ of EDTA-Sarcosyl was then added and the solution centrifuged at $4,000 \mathrm{rpm}$ for 15 minutes. The supernatant was then poured off and thus the residue of calcium was removed. Then $7 \mathrm{ml}$ of EDTA-Sarcosyl (pH 8.0) and $200 \mu$ Proteinase $\mathrm{K}(20 \mu \mathrm{g} / \mathrm{ml})$ were added and the sample mixed thoroughly by vortexing. Incubation at $56^{\circ} \mathrm{C}$ followed and continued until dissolution of the solid particles was complete and then $7 \mathrm{ml}$ of phenol:chlorophorm:isoamylalcohol, 25:24:1 was added. The sample was then gently shaken and centrifuged at 4,000 rpm for 15 minutes, the upper aqueous phase containing DNA was transferred to another clean tube and this organic extraction procedure was repeated twice more. To concentrate the DNA to a final volume of $150 \mu 1$, Centricon columns were used (Centricon YM, Centrifugal Filter Devices, MILLIPORE).

\section{Isolation of DNA from buccal swabs of recent greyhounds}

DNA from the buccal swabs of 15 recent dogs was isolated with a 5\% suspension of Chelex (Walsh et al. 1991).

\section{Amplification of mtDNA from isolates of bone and buccal swabs}

Oligonucleotide primers used for amplification and sequencing of the canid mtDNA CR were created using a dog reference sequence (GenBank accession no. U96639). Convention nomenclature was used: the primer name indicates the position of the $5^{\prime}$ base. Forward primers were F15.719 (5'-GTAATGTCCCTCTTCTCGCT-3') and F16.431 (5'-CACGCGCGTAAGACATTAAG-3'). Reverse primers were R16.114 (5'-CCTGAAACCATTGACTGAATAG-3') and R42 (5'-GGCATTTTCAGTGCCTTGCTT-3') (Gundry et al. 2007, see Text-fig. 2 for the position of primers). Lyophilized primers (Generi Biotech, Czech Republic) were suspended to produce a concentration of $10 \mathrm{mM}$ in $\mathrm{dd}_{2} \mathrm{O}$.

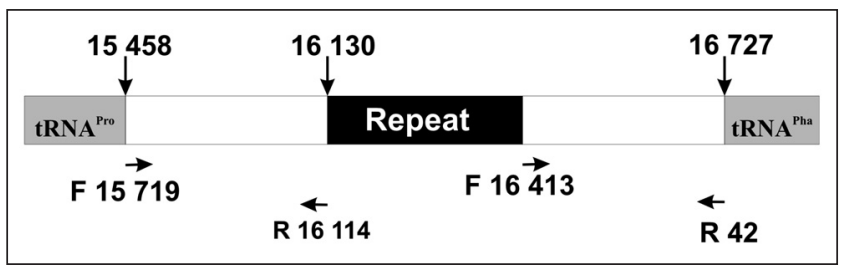

Text-fig. 2. Scheme of position of primers on mtDNA D-loop (adopted from Gundry et al. 2007).
$5 \mu$ of total genomic dog DNA was used to amplify the chosen regions in a $20 \mu \mathrm{l}$ reaction volume containing the following reagents: $200 \mathrm{mM}$ of each dNTP (Applied Biosystems), $0.6 \mathrm{mM}$ each of primers F15,412; F16,431; R16,114 and R42, 5U AmpliTaq Gold DNA polymerase (Applied Biosystems) and 1× GeneAmpTM PCR Buffer containing $1.5 \mathrm{mM} \mathrm{MgCl}$ (Applied Biosystems). Amplifications were conducted in a GeneAmp 9700 PCR System thermal cycler (Applied Biosystems), and consisted of denaturation for $11 \mathrm{~min}$. at $95^{\circ} \mathrm{C}$, followed by 30 cycles of $1 \mathrm{~min}$. at $94{ }^{\circ} \mathrm{C}, 1 \mathrm{~min}$. at $58.9^{\circ} \mathrm{C}$, and $2 \mathrm{~min}$. at $72^{\circ} \mathrm{C}$, plus final incubation for $60 \mathrm{~min}$. at $60^{\circ} \mathrm{C}$. Genomic DNA of recent dog was used as a positive control and sterile $\mathrm{ddH}_{2} \mathrm{O}$ as a negative control. A total of $2 \mu \mathrm{l}$ of each sample was visualised on $2 \%$ Agarose gel, $5 \mathrm{~V} / \mathrm{cm} / 30 \mathrm{~min}$. (Text-fig. 3 ).

DNA isolation from the test objects were performed at an accredited workplace of OKTE (Department of Criminalistic Technique and Expertises), amplification and subsequent sequencing in accredited laboratories GeneProof, as and sequencing of mtDNA from bones was repeated $3 \times$ for each isolate with the same result. Isolation of mtDNA from the bones was repeated six months later in a laboratory which is normally working only with human DNA. Isolation of mtDNA from the Ulster greyhound was performed separately and after isolation of DNA from bone thus preventing any contamination.

\section{Sequencing of mtDNA}

Cycle sequencing was performed using a Terminator Ready Reaction Mix (Applied Biosystems) and BigDye Sequencing Buffer (Applied Biosystems) according to the manufacturer's instructions. The sequencing primers were the same as those listed above. The sequencing reaction (test solution containing $7 \mu \mathrm{l}$ of the amplified DNA, $3.5 \mu \mathrm{l}$ primer [at $1 \mathrm{mM}$ ] and $9.5 \mu \mathrm{l}$ Ready Reaction mix) was carried out in a GeneAmp 9700 PCR System thermal cycler and consisted of denaturation for $1 \mathrm{~min}$. at $96{ }^{\circ} \mathrm{C}$, followed by 25 cycles of $10 \mathrm{sec}$. at $96{ }^{\circ} \mathrm{C}, 5 \mathrm{sec}$. at $60{ }^{\circ} \mathrm{C}$, and $4 \mathrm{~min}$. at $60{ }^{\circ} \mathrm{C}$, followed by a holding period at $12{ }^{\circ} \mathrm{C}$ until the next step.

\section{Results}

We amplified DNA from two parallel isolates of the same bone and we obtained two identical 395 bp sequences with primer pair A (F15.719 and R16.114) and two identical 338 bp sequences with primer pair B (F16.431 and R42) (Text-fig. 3). The blank extraction control was negative. To verify that we had worked with ancient DNA we used primer pairs: F15.719, R16.169 (450 bp), R16.269 (550 bp) and R16.519 (800 bp) that did not yield any amplicon, which is considered a signature of degraded, presumably ancient DNA (Text-fig. 4). We compared the obtained sequences with the corresponding sequences of recent dogs published by Gundry et al. (2007).

In the next step we compared our sequences from ancient bone and sequences of a recent greyhound from N. Ireland. The first sequences were obtained by primer pair A (Text-fig. 5) the second sequences by primer pair B (Text-fig. 6). 


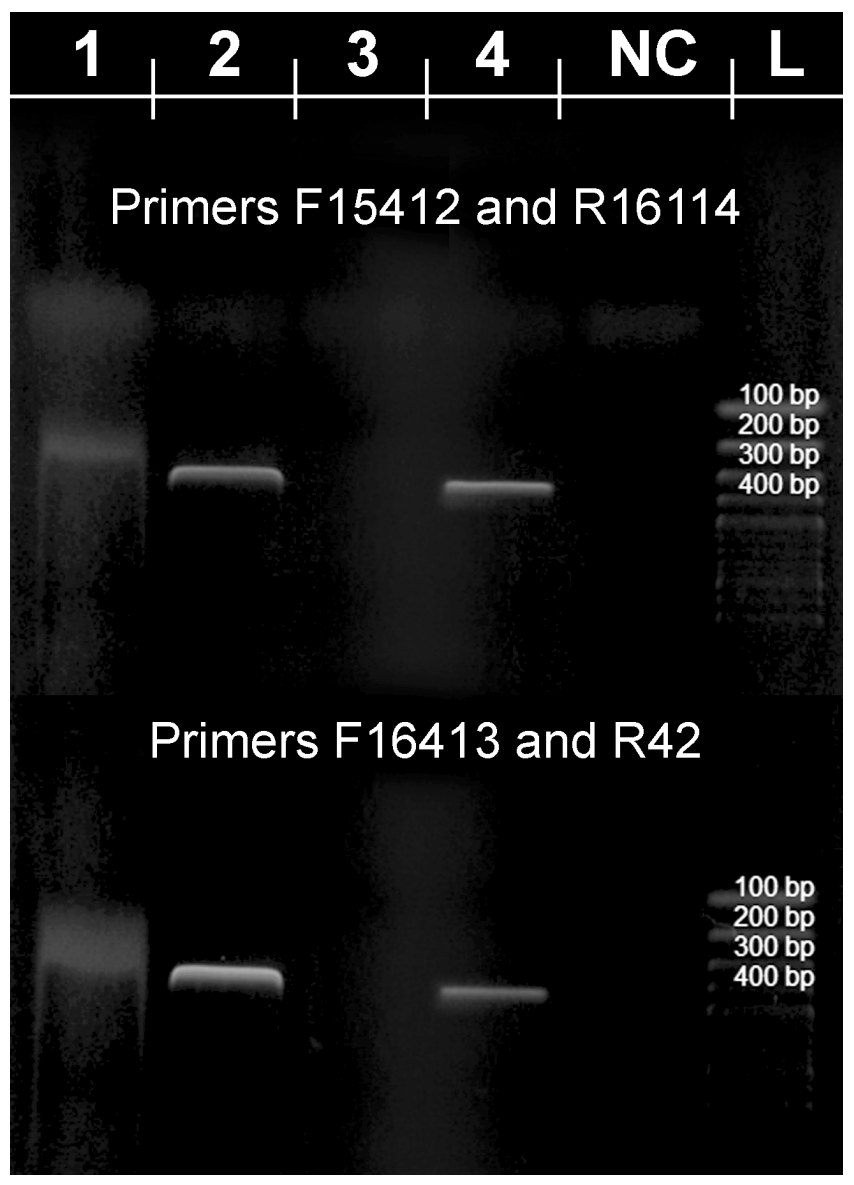

Text-fig. 3. Electrophoresis after amplification: Electrophoretical analysis of mitochondrial DNA from two parallel bone samples. In the upper part of the figure, are mtDNA sequences amplified by primer pair A (F15.412 and R16.114 (395 bp)), and in the lower part by primer pair B (F16.431 and R42 (338 bp)). Lane 1 and 3 - undiluted samples, 2 and 4 - 10× diluted samples, $\mathrm{NC}$ - negative control, $\mathrm{L}-100$ bp DNA ladder (band size from $100 \mathrm{bp}$ to $1500 \mathrm{bp}$ ).

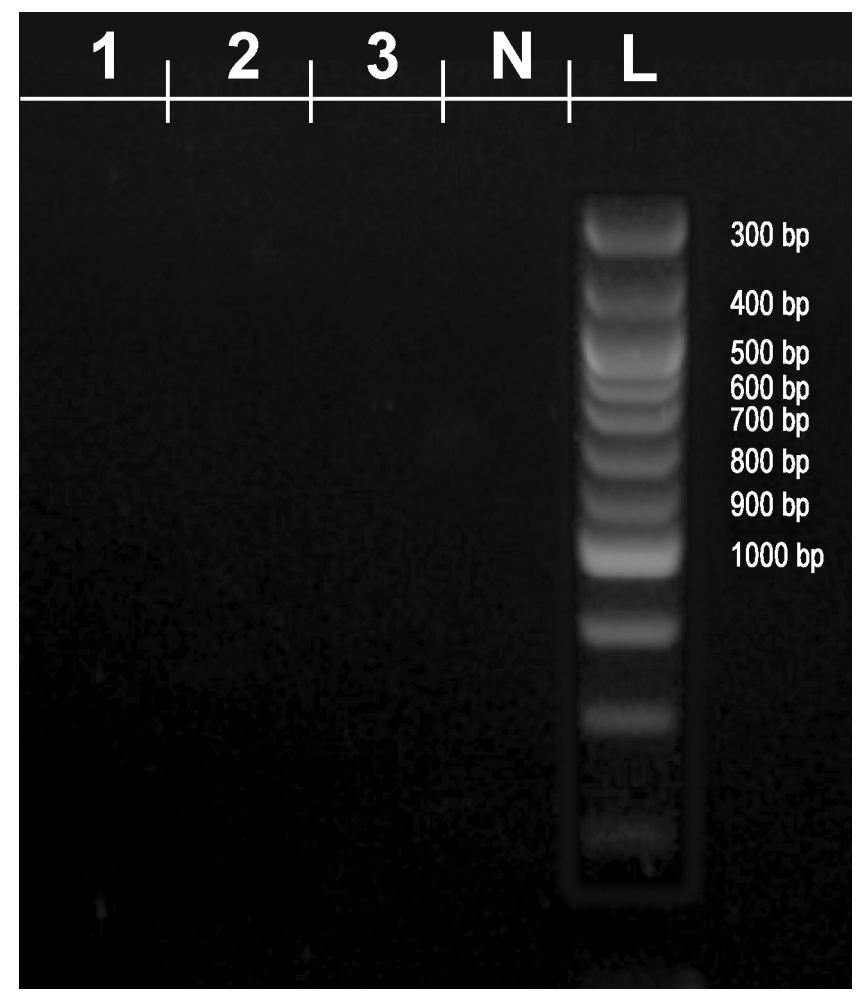

Text-fig. 4. Electrophoresis after amplification: Electrophoretical analysis of mitochondrial DNA. mtDNA sequences were amplified by primers F15.412 and R16.169 (450 bp), R16.269 (550 bp), R16.519 (800 bp). Lane 1 are primers F15.412 + R16.169, lane 2 primers F15.412 + R16.269, lane 3 primers F15.412 + R16.519, NC - negative control - water, $\mathrm{L}-100$ bp DNA ladder (band size from $100 \mathrm{bp}$ to $1500 \mathrm{bp}$ ).

Text-fig. 7 is the comparison of sequences of mtDNA from mtDNA from BLAST and mtDNA from ancient bone with primer R42. In Table 1 and 2 we can see samples from 16

\begin{tabular}{|c|c|c|c|}
\hline Recent & 1 & $\begin{array}{l}\text { СтTTATTTACTCCAATCCTACTAATTCTCGCAAATGGGACATCTCGATGGACTAATGACT } \\
||||||||||||||||||||||||||||||||||||||||||||||||||||||||||||||||||\end{array}$ & 60 \\
\hline Bone & 1 & СтTTATTTACTCCAATCCTACTAATTCTCGCAAATGGGACATCTCGATGGACTAATGACT & 60 \\
\hline Recent & 61 & $\begin{array}{l}\text { AATCAGCCCATGATCACACATAACTGTGGTGTCATGCATCTGGTATCTTTTAATTTTTAg } \\
|||||||||||||||||||||||||||||||||||||||||||||||||||||||||||||||||| \mid\end{array}$ & 120 \\
\hline Bone & 61 & AАTCAGCCCATGATCACACATAACTGTGGTGTCATGCATCTGGTATCTTTTAATTTTTAG & 120 \\
\hline Recent & 121 & $\begin{array}{l}\text { ggggggAATCTGCTATCACTCATCTACGACCGCAACGGCACTAACTCTAACTTATCTTCT } \\
|||||||||||||||||||||||||||||||||||||||||||||||||||||||||||||||| \mid\end{array}$ & 180 \\
\hline Bone & 121 & GGGGGGAATCTGCTATCACTCATCTACGACCGCAACGGCACTAACTCTAACTTATCTTCT & 180 \\
\hline Recent & 181 & $\begin{array}{l}\text { GCTCTCAGGGAATATGCCCGTCGCGGCCCTAATGCAGTCAAATAACTTGTAGCTGGACTT } \\
|||||||||||||||||||||||||||||||||||||||||||||||||||||||||||||||||| \mid\end{array}$ & 240 \\
\hline Bone & 181 & GCTCTCAGGGAATATGCCCGTCGCGGCCCTAATGCAGTCAAATAACTTGTAGCTGGACTT & 240 \\
\hline Recent & 241 & $\begin{array}{l}\text { АТTСАТТАТСATTTATCAACTCACGCATAAAATCAAGG } \\
|||||||||||||||||||||||||||||||||||||||||| \mid\end{array}$ & \\
\hline Bone & 241 & АТTСАTTATСАTTTATСAАСTCACGСATAAAATCAAGG & \\
\hline
\end{tabular}

Text-fig. 5. Multiple sequence alignment of mtDNA from ancient bone and recent greyhound, (Gundry et al. 2007) primer pair A - F15.719 and R16.114. 


\begin{tabular}{|c|c|c|c|}
\hline Recent & 1 & 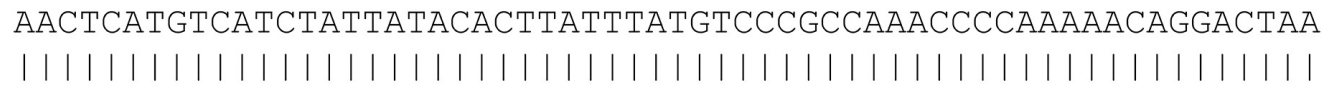 & 60 \\
\hline Bone & 3 & ААСTCATGTCATCTATTATACACTTATTTATGTCCCGCCAAACCCCAAAAACAGGACTAA & 62 \\
\hline Recent & 61 & 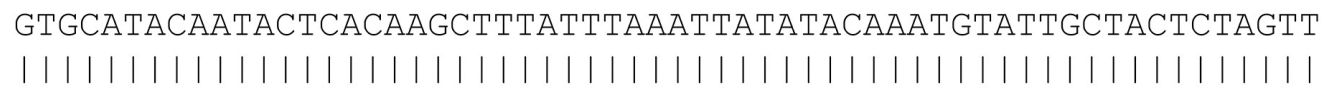 & 120 \\
\hline Bone & 63 & GTGCATACAATACTCACAAGCTTTATTTAAATTATATACAAATGTATTGCTACTCTAGTT & 122 \\
\hline Recent & 121 & 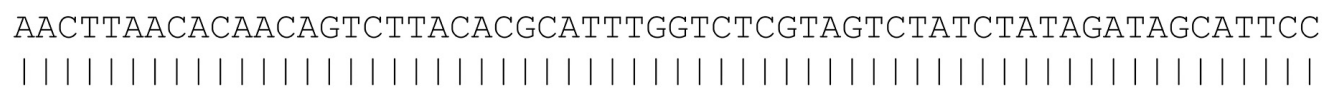 & 180 \\
\hline Bone & 123 & AACTTAACACAACAGTCTTACACGCATTTGGTCTCGTAGTCTATCTATAGATAGCATTCC & 182 \\
\hline Recent & 181 & 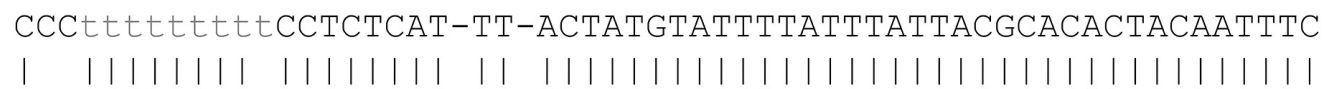 & 238 \\
\hline Bone & 183 & С--тTтTтTтCССТСТCATATTтACTATGTATTTTATTTATTACGCACACTACAATTTC & 240 \\
\hline Recent & 239 & $\begin{array}{l}\text { AGTATAA } 244 \\
\text { ||||||| }\end{array}$ & \\
\hline Bone & 241 & AGTATA & \\
\hline
\end{tabular}

Text-fig. 6. Comparison of overlapping sequences of mtDNA from recent greyhound and mtDNA from ancient bone - primer pair B F16.431 and R42.

\begin{tabular}{|c|c|c|c|}
\hline Query & 16461 & CAAAccсccettaccсCсCGTAACTCATGTCATCTATTATACACTTATTTATGTCCCGC & 16520 \\
\hline & & ||||||||||||||||||||||||||||||||||||$|\quad|||||||||||$ & \\
\hline Sbjet & 276 & САААССССYСТTMYСССYCGTAАACTSAWGTCATCTATTATACWMWWATTTATGKCCCGY & 217 \\
\hline
\end{tabular}

Text-fig. 7. Comparison of overlapping sequences of mtDNA from mtDNA from BLAST and mtDNA from ancient bone - primer R42 (query is mtDNA from BLAST and subject is mtDNA from bone), position 16,480 is colour-coded.

greyhounds. As a reference sequence the mtDNA from bone compared with the mtDNA of 15 recent greyhounds in the Czech Republic is used.

\section{Discussion}

The evidence of a greyhound in the Slavonic and later in the Great Moravian period within the Czech territory is extraordinary, and it represents the first of this kind ever found in the Czech lands.

We authenticated the ancient DNA using the possibility to amplify long mtDNA fragments (with a length of $450 \mathrm{bp}$, $550 \mathrm{bp}$ and $800 \mathrm{bp}$ ) (Herrmann and Hummel 1994, Hummel 2003). By comparing the sequences from the ancient bone and from the buccal swab with the help of BLAST (Basic Local Alignment Search Tool), we confirmed that the

Table 1. Comparison of mtDNA sequences from recent greyhounds and mtDNA from bone with primers F15.719 and R16.114. Mutations are described in the upper line, samples are marked as 1 to 15.

\begin{tabular}{|c|c|c|c|c|c|c|c|c|c|c|c|c|}
\hline $\begin{array}{l}\text { F15719 and } \\
\text { R16114 }\end{array}$ & *A/AA & & & ${ }_{\mathrm{G}}^{* *} \mathrm{C} / \mathrm{T},+\mathrm{GA}, \mathrm{T} / \mathrm{C},-$ & $T / C$ & & & & $+\mathrm{A}$ & & & \\
\hline Bone & TATCATGAAA & СTATACCTGG & СATCTGGTTC & $\begin{array}{l}\text { TTACTTCAGG } \\
\end{array}$ & GCCATAACTT & TATTTACTCC & AATCCTACTA & ATTCTCGCAA & ATGGGACATC & TCGATGGACT & AATGACTAA & TCAGCCCATG \\
\hline 1 & TATCATGAA & CTATACCTGG & CATCTGGTTC & TTACCTCAGG & GCCATAACTT & TATTTACTCC & AATCCTACTA & ATTCTCGCAA & ATGGGACATC & TCGATGGACT & AATGACTAA & TCAGCCCATG \\
\hline 2 & TATCATGAA & CTATACCTGG & CATCTGGTTC & TTACCTCAGG & GCCATAACTC & TATTTACTCC & AATCCTACTA & ATTCTCGCAA & ATAGGGACATC & TCGATGGACT & AATGACTAA & TCAGCCCATG \\
\hline 3 & TATCATGA & CTATACCTGG & CATCTGGTTC & TTACCTCAGG & GCCATAACTC & TATTTACTCC & AATCCTACTA & ATTCTCGCAA & ATAGGGACATC & TCGATGGACT & AATGACTAA & TCAGCCCATG \\
\hline 4 & TATCATGAA & CTATACCTGG & CATCTGGTTC & TTACCTCAGG & GCCATAACTC & TATTTACTCC & AATCCTACTA & ATTCTCGCAA & ATGGGACATC & TCGATGGACT & AATGACTAA & TCAGCCCATG \\
\hline 5 & TATCATGA & СTATACCTGG & CATCTGGTTC & TTACTTCAGG & GCCATAACTT & TATTTACTCC & AATCCTACTA & ATTCTCGCAA & ATGGGACATC & TCGATGGACT & AATGACTAA & TCAGCCCATG \\
\hline 6 & TATCATGA & CTATACCTGG & CATCTGGTTC & TTACCTCAGG & GCCATAACTC & TATTTACTCC & AATCCTACTA & ATTCTCGCAA & ATAGGACATC & TCGATGGACT & AATGACTAA & TCAGCCCATG \\
\hline 8 & TATCATGA & CTATACCTGG & CATCTGGTTC & TTACTTCAGAGG & GCCATAACTT & TATTTACTCC & AATCCTACTA & ATTCTCGCAA & ATAGGGACATC & TCGATGGACT & AATGACTAA & TCAGCCCATG \\
\hline 9 & TATCATGA & CTATACCTGG & CATCTGGTTC & TTACTTCAGG & GCCATAACTT & TATTTACTCC & AATCCTACTA & ATTCTCGCAA & ATGGGACATC & TCGATGGACT & AATGACTAA & TCAGCCCATG \\
\hline 10 & TATCATGA & CTATACCTGG & CATCTGGTTC & TTACTTCAGG & GCCATAACTT & TATTTACTCC & AATCCTACTA & ATTCTCGCAA & ATGGGACATC & TCGATGGACT & AATGACTAA & TCAGCCCATG \\
\hline 11 & TATCATGAA & CTATACCTGG & CATCTGGTTC & TTACCTCAGG & GCCATAACTC & TATTTACTCC & AATCCTACTA & ATTCTCGCAA & ATGGGACATC & TCGATGGACT & AATGACTAA & TCAGCCCATG \\
\hline 12 & TATCATGA & CTATACCTGG & CATCTGGTTC & TTACCTCAGAG & GCCATAACTC & TATTTACTCC & AATCCTACTA & ATTCTCGCAA & ATGGGACATC & TCGATGGACT & AATGACTAA & TCAGCCCATG \\
\hline 13 & TATCATGAA & CTATACCTGG & CATCTGGTTC & TTACTTCAGG & GCCATAACTT & TATTTACTCC & AATCCTACTA & ATTCTCGCAA & ATGGGACATC & TCGATGGACT & AATGACTAA & TCAGCCCATG \\
\hline 14 & TATCATGAA & CTATACCTGG & CATCTGGTTC & TTACTTCAGG & GCCATAACTT & TATTTACTCC & AATCCTACTA & ATTCTCGCAA & ATGGGACATC & TCGATGGACT & AATGACTAA & TCAGCCCATG \\
\hline
\end{tabular}


Table 1. [continued]

\begin{tabular}{|c|c|c|c|c|c|c|c|c|c|}
\hline $\begin{array}{l}\begin{array}{l}\text { F15719 and } \\
\text { R16114 }\end{array} \\
\text { R }\end{array}$ & & $\mathrm{T} / \mathrm{C}$ & & & & & $\mathrm{A} / \mathrm{G}$ & & \\
\hline bone & GCGGCCCTAA & TGCAGTCAAA & TAACTTGTAG & CTGGAGTTAT & TCATTATCAT & TTATCAACTC & ACGCATAAA & ATCAAGGTG & CTATTCAGT \\
\hline 1 & GCGGCCCTAA & TGCAGTCAAA & TAACTTGTAG & CTGGACTTAT & TCATTATCAT & TTATCAACTC & ACGCATAAG & ATCAAGGTG & CTATTCAGT \\
\hline 2 & GCGGCCCTAA & TGCAGTCAAA & TAACTTGTAG & CTGGACTTAT & TCATTATCAT & TTATCAACTC & ACGCATAAG & ATCAAGGTG & CTATTCAGT \\
\hline 3 & GCGGCCCTAA & TGCAGTCAAA & TAACTTGTAG & CTGGACTTAT & TCATTATCAT & TTATCAACTC & ACGCATAAG & ATCAAGGTG & $\begin{array}{l}\text { CTATTCAGT } \\
\end{array}$ \\
\hline 4 & GCGGCCCTAA & TGCAGTCAAA & TAACTTGTAG & CTGGACTTAT & TCATTATCAT & TTATCAACTC & ACGCATAAG & ATCAAGGTG & CTATTCAGT \\
\hline 5 & GCGGCCCTAA & CGCAGTCAAA & TAACTTGTA & CTGGACTTAT & TCATTATCAT & TTATCAACTC & ACGCATAAA & ATCAAGGTG & CTATTCAGT \\
\hline 6 & GCGGCCCTAA & TGCAGTCAAA & TAACCTGTAG & CTGGACTTAT & TCATTATCAT & TTATCAACTC & ACGCATAAA & ATCAAGGTG & CTATTCAGT \\
\hline 7 & GCGGCCCTAA & TGCAGTCAAA & TAACTTGTAG & CTGGACTTAT & TCATTATCAT & TTATCAACTC & ACGCATAAA & ATCAAGGTG & CTATTCAGT \\
\hline 8 & GCGGCCCTAA & CGCAGTCAAA & TAACTTGTAG & CTGGACTTAT & TCATTATCAT & TTATCAACTC & ACGCATAAA & ATCAAGGTG & CTATTCAGT \\
\hline 9 & GCGGCCCTAA & CGCAGTCAAA & TAACTTGTAG & CTGGACTTAT & TCATTATCAT & $\begin{array}{l}\text { TTATCAACTC } \\
\end{array}$ & ACGCATAAA & ATCAAGGTG & $\begin{array}{l}\text { CTATTCAGT } \\
\end{array}$ \\
\hline 10 & GCGGCCCTAA & CGCAGTCAAA & TAACTTGTAG & CTGGACTTAT & TCATTATCAT & TTATCAACTC & ACGCATAAA & ATCAAGGTG & CTATTCAGT \\
\hline 11 & GCGGCCCTAA & TGCAGTCAAA & TAACTTGTAG & CTGGACTTAT & TCATTATCAT & TTATCAACTC & ACGCATAAG & ATCAAGGTG & CTATTCAGT \\
\hline 12 & GCGGCCCTAA & TGCAGTCAAA & TAACTTGTAG & $\begin{array}{l}\text { CTGGACTTAT } \\
\end{array}$ & TCATTATCAT & TTATCAACTC & ACGCATAAG & ATCAAGGTG & $\begin{array}{l}\text { CTATTCAGT } \\
\end{array}$ \\
\hline 13 & GCGGCCCTAA & CGCAGTCAAA & TAACTTGTAG & CTGGACTTAT & TCATTATCAT & TTATCAACTC & ACGCATAAA & ATCAAGGTG & CTATTCAGT \\
\hline 14 & GCGGCCCTAA & TGCAGTCAAA & TAACTTGTAG & CTGGACTTAT & TCATTATCAT & TTATCAACTC & ACGCATAAA & ATCAAGGTG & CTATTCAGT \\
\hline 15 & GCGGCCCTAA & TGCAGTCAAA & TAACTTGTAG & CTGGACTTAT & TCATTATCAT & TTATCAACTC & ACGCATAAA & ATCAAGGTG & CTATTCAGT \\
\hline
\end{tabular}

Table 2. Comparison of mtDNA sequences from recent greyhounds and mtDNA from bone with primers F16.431 and R42, mutations are described in the upper line, samples are marked as 1 to 15.

\begin{tabular}{|c|c|c|c|c|c|c|c|c|c|c|c|}
\hline $\begin{array}{l}\text { F16431 } \\
\text { and R42 }\end{array}$ & & & & & & & & & & $\mathrm{T} / \mathrm{C}$ & $+\mathrm{A}$ \\
\hline bone & AACTCATGTC & ATCTATTATA & CACTTATTTA & TGTCCCGCCA & AACCCCAAAA & ACAGGACTA & AGTGCATACA & ATACTCACAA & GCTTTATTTA & AATTATATAC & AAATGTATTG \\
\hline 1 & AACTCATGTC & ATCTATTATA & CACTTATTTA & TGTCCCGCCA & AACCCCAAAA & ACAGGACTA & AGTGCATACA & ATACTCACAA & GCTTTATTTA & AATTATATAC & AAATGTATTG \\
\hline 2 & AACTCATGTC & ATCTATTATA & CACTTATTTA & TGTCCCGCCA & AACCCCAAAA & ACAGGACTA & AGTGCATACA & ATACTCACAA & GCTTTATTTA & AATTATATAC & AAATGTATTG \\
\hline 3 & AACTCATGTC & ATCTATTATA & CACTTATTTA & TGTCCCGCCA & AACCCCAAAA & ACAGGACTA & AGTGCATACA & ATACTCACAA & GCTTTATTTA & AATTATATAC & AAATGTATTG \\
\hline 4 & AACTCATGTC & ATCTATTATA & САCTTATTTA & TGTCCCGCCA & AACCCCAAAA & ACAGGACTA & AGTGCATACA & ATACTCACAA & GCTTTATTTA & AATTATATAC & AAATGTATTAG \\
\hline 5 & AACTCATGTC & ATCTATTATA & CACTTATTTA & TGTCCCGCCA & AACCCCAAAA & ACAGGACTA & AGTGCATACA & ATACTCACAA & GCTTTATTTA & AATTATATAC & AAATGTATTG \\
\hline 6 & AACTCATGTC & ATCTATTATA & CACTTATTTA & TGTCCCGCCA & AACCCCAAAA & ACAGGACTA & AGTGCATACA & CACTCACAA & GCTTTATTTA & AATCATATAC & AAATGTATTG \\
\hline 7 & AACTCATGTC & ATCTATTATA & CACTTATTTA & TGTCCCGCCA & AACCCCAAAA & ACAGGACTA & AGTGCATACA & ATACTCACAA & GCTTTATTTA & AATTATATAC & AAATGTATTG \\
\hline 8 & AACTCATGTC & ATCTATTATA & CACTTATTTA & TGTCCCGCCA & AACCCCAAAA & ACAGGACTA & AGTGCATACA & ATACTCACAA & GCTTTATTTA & AATTATATAC & AAATGTATTG \\
\hline 9 & AACTCATGTC & ATCTATTATA & CACTTATTTA & TGTCCCGCCA & AACCCCAAAA & ACAGGACTA & AGTGCATACA & ATACTCACAA & GCTTTATTTA & AATTATATAC & AAATGTATTG \\
\hline 10 & AACTCATGTC & ATCTATTATA & CACTTATTTA & TGTCCCGCCA & AACCCCAAAA & ACAGGACTA & AGTGCATACA & ATACTCACAA & GCTTTATTTA & AATTATATAC & AAATGTATTG \\
\hline 11 & AACTCATGTC & ATCTATTATA & CACTTATTTA & TGTCCCGCCA & AACCCCAAAA & ACAGGACTA & AGTGCATACA & ATACTCACAA & GCTTTATTTA & AATTATATAC & AAATGTATTG \\
\hline 12 & AACTCATGTC & ATCTATTATA & CACTTATTTA & TGTCCCGCCA & AACCCCAAAA & ACAGGACTA & AGTGCATACA & ATACTCACAA & GCTTTATTTA & AATTATATAC & AAATGTATTG \\
\hline 14 & AACTCATGTC & ATCTATTATA & САCTTATTTA & TGTCCCGCCA & AACCCCAAAA & ACAGGACTA & AGTGCATACA & ATACTCACAA & GCTTTATTTA & AATTATATAC & AAATGTATTG \\
\hline 15 & AACTCATGTC & ATCTATTATA & CACTTATTTA & TGTCCCGCCA & AACCCCAAAA & ACAGGACTA & AGTGCATACA & ATACTCACAA & GCTTTATTTA & AATTATATAC & AAATGTATTG \\
\hline
\end{tabular}

Table 2. [continued]

\begin{tabular}{|c|c|c|c|c|c|c|c|c|c|c|c|c|c|c|}
\hline $\begin{array}{l}\text { F16431 } \\
\text { and R42 }\end{array}$ & & & & & & & $-\mathrm{C}$ & $-1+\mathrm{T}$ & $\begin{array}{c}* * * \\
* \mathrm{TTT}^{2} \mathrm{CCC},+\mathrm{C} \\
+\mathrm{TA}\end{array}$ & & & & & \\
\hline bone & IACTCTAGT & ACTTAACA & ACAGTCTT & CACGCATTT & GTCTCGTAG & TATCTATA & ATAGCATTC & CCCCHtttt & tttCCTCTCA & TTTACTATGT & ITTTTATTTA & TTACGCACAC & TACAATTTCA & GTATAA \\
\hline 1 & СTACTCTAGT & TAACTTAACA & CAACAGTCTT & ACACGCATTT & GGTCTCGTAG & TCTATCTATA & GATAGCATTC & ССтTтTTT & ТTTCСТCTCATA & TTTACTATGT & ATTTTATTTA & TTACGCACAC & TACAATTTCA & GTATAA \\
\hline 2 & СTACTCTAGT & TAACTTAACA & CAACAGTCTT & ACACGCATTT & GGTCTCGTAG & TCTATCTATA & GATAGCATTC & CСTTTTT & TTТСССТСССТA & TTTACTATGT & ATTTTATTTA & TTACGCACAC & TACAATTTCA & GTATAA \\
\hline 3 & CTACTCTAGT & TAACTTAACA & AACAGTCTT & ACACGCATTT & GGTCTCGTAG & TCTATCTATA & GATAGCATTC & CCTTTTTT & TTTCCTCTCATA & TTTACTATGT & ATTTTATTTA & TTACGCACAC & TACAATTTCA & $\begin{array}{l}\text { GTATAA } \\
\end{array}$ \\
\hline 4 & CTACTCTAGT & TAACTTAACA & CAACAGTCTT & ACACGCATTT & GGTCTCGTAG & TCTATCTATA & GATAGCATTC & сCтTTTTT & TTTCCTCTCATA & TTTACTATGT & ATTTTATTTA & TTACGCACAC & TACAATTTCA & GTATAA \\
\hline 5 & CTACTCTAGT & TAACTTAACA & CAACAGTCTT & ACACGCATTT & GGTCTCGTAG & TCTATCTATA & GATAGCATTC & CCTTTTT & ТTТСССТСТCATA & TTTACTATGT & ATTTTATTTA & TTACGCACAC & TACAATTTCA & GTATAA \\
\hline 6 & CTACTCTAGT & TAACTTAACA & CAACAGTCTT & ACACGCATTT & GGTCTCGTAG & TCTATCTATA & GATAGCATTC & CCTTTTTT & СССССТСТCATA & TTTACTATGT & ATTTTATTTA & TTACGCACAC & TACAATTTCA & GTATAA \\
\hline 8 & CTACTCTAGT & TAACTTAACA & CAACAGTCTT & ACACGCATTT & GGTCTCGTAG & TCTATCTATA & GATAGCATTC & CСTTTTT & TTTCССТCTCATA & TTTACTATGT & ATTTTATTTA & TTACGCACAC & TACAATTTCA & GTATAA \\
\hline 9 & CTACTCTAGT & TAACTTAACA & CAACAGTCTT & ACACGCATTT & GGTCTCGTAG & TCTATCTATA & GATAGCATTC & CСTTTTT & TTТCССТСТCATA & TTTACTATGT & ATTTTATTTA & TTACGCACAC & TACAATTTCA & GTATAA \\
\hline 10 & CTACTCTAGT & TAACTTAACA & CAACAGTCTT & ACACGCATTT & GGTCTCGTAG & TCTATCTATA & $\begin{array}{l}\text { GATAGCATT } \\
\end{array}$ & ССтTTTT & TTТСССТСТCATA & TTTACTATGT & ATTTTATTTA & TTACGCACAC & TACAATTTCA & GTATAA \\
\hline 11 & CTACTCTAGT & TAACTTAACA & CAACAGTCTT & ACACGCATTT & GGTCTCGTAG & TCTATCTATA & GATAGCATTC & CCTTTTTT & TTTCCTCTCATA & TTTACTATGT & ATTTTATTTA & TTACGCACAC & TACAATTTCA & GTATAA \\
\hline 12 & СTACTCTAGT & TAACTTAACA & CAACAGTCTT & ACACGCATTT & GGTCTCGTAG & TCTATCTATA & GATAGCATTC & сСтTTTTT & TTTCCTCTCATA & TTTACTATGT & ATTTTATTTA & TTACGCACAC & TACAATTTCA & GTATAA \\
\hline 14 & IACTCTAGT & ACCTTAACA & AACAGTCTT & ACACGCATTT & GGTCTCGTAG & TCTATCTATA & GATAGCATTC & CСТTTTT & TTTCCCTCTCATA & TTTACTATGT & ATTTTATTTA & & TACAATTTCA & GTATAA \\
\hline 15 & СТACTC & $\mathrm{ACA}$ & TCTT & CATTT & GTAG & TATA & ATAGCATTC & ССТTTTT & ТТТСССТСТСАТА & TTTACTATGT & ATTTTATTTA & TTACGCACAC & TACAATTTCA & GTATAA \\
\hline
\end{tabular}

sequences of mitochondrial DNA came from a dog (99\% identical). By comparing our data with Gundry et al.'s (2007), we confirmed that our PCR amplicons with primer pairs $\mathrm{A}$ and $\mathrm{B}$ are located on canine mtDNA.

The sequences from both the recent greyhound from $\mathrm{N}$. Ireland and the ancient bone obtained by using PCR with primer pair A were identical. However, the sequences amplified with primer pair B contained four deletions and one substitution (Text-fig. 6). We suggest that the first two deletions are at positions 184 and 185, the third at 203 and the fourth at position 204 in the recent dog sequence in comparison with the ancient bone sequence. One substitution is at position 194 where nucleotide $\mathrm{C}$ is replaced by T. These differences probably arose during 11 centuries of dog breeding (Herrmann and Hummel 1994).

We compared our sequences with the reference database presented by Gundry et al. (2007) which contains dog mtDNA CR sequences, variable SNPs, and haplotypes of 125 American domestic dog breeds and three wild canids. In particular we compared the SNPs at positions which are 
specific for greyhounds and we discovered that one greyhound SNP is different. This part of bone sequence from position 16,461 to 16,490 is $3^{\prime}$-CAAACCCCCC TTACCCCCG TAAACTCATG-5'. At position 16,480 is nucleotide A which is specific for the greyhound. Nucleotide $G$ at this position is specific for the Yorkshire Terrier or American Eskimo Dog and American Spitz, which are in the same group. All sequences established with primers set $\mathrm{B}$, which determined the area 16,461 to 16,490 had nucleotide $G$ at position 16,480 . These findings seem to be a misleading result but we should note that our comparison was between different dogs from two different areas (Europe and North America). In the article was described only one greyhound sequence and this is probably the reason for the discrepancy (Zajc et al. 1997). Our recent greyhounds are demonstrably greyhounds and we found guanine at position 16,480. The same nucleotide was located at the same position in the sequence of the ancient bone found at the hillfort Chotěbuz-Podobora. After the previous comparison of ancient mtDNA with recent mtDNA from the N. Ireland greyhound we found that they are so similar that we are persuaded our ancient biological material comes from a greyhound and in view of the impressive history of this breed, there was a high probability that it was unthinkable to interbreed greyhounds with any other breed of dogs.

Furthermore, we compared the sequences of mtDNA from bone with sequences from recent greyhounds from the dog exhibition in Brno 2010, as can be seen in Tables 1 and 2. Compared to the ancient mtDNA deletions, insertions and substitutions were evident. The differences are shown in Tables 1 and 2. Some of them are marked by one, two or three stars because they appear interesting from an evolutionary point of view. We presume that for example a deletion of one or two A nucleotides (in the table it is shown as *) is the result of molecular evolution and more A nucleotides signify that the individual is evolutionarilly older with respect to the mtDNA. Sample 8 is MAYSUN Bohemia Genao, whose grandfather was a Moroccan Sloughi, and is the only individual in which we observed the addition of a group of GA nucleotides (shown as **). The column with $* * *$ is also very variable. All recent dogs have an added pair of TA nucleotides in their mtDNA. The sloughi (sample 6) has a triplet of $\mathrm{T}$ substituted by a triplet of $\mathrm{C}$. All changes found constitute the passage of time. The more changes compared to ancient DNA, the younger the specimen is. With respect to the foundations of paleogenetics, we can talk about a dog's "Eves", the rare changes signify different molecular "Eves", different places of development (Horai et al. 1995).

According to historical sources, greyhounds are a very ancient breed that has been prized by nobility for thousands of years. They can reach speeds of over $65 \mathrm{~km} / \mathrm{h}$ being thus the fastest dog breed in the world. The true origin of the greyhound is unsure, but drawings of findings from the Çatal Hüyük site in Turkey (6000 BC), the finding of a greyhound-like dog in a funereal vase in the town of Fusa in Iran $(4200 \mathrm{BC})$ or in rock art in Tassili (dated at 5000-2000 BC) indicate that the greyhound is indeed one of the oldest breeds of dog (Bazin 1959, Brentjes 1969, Branigan 2004).

The greyhound-like dog came to the ChotěbuzPodobora site almost certainly as an exclusive article from the territory of present-day Poland, probably as a gift to the local lord, or as loot. The presence of the greyhound-like dog coincides with other exceptional findings from Chotěbuz-Podobora which are dated from the second half of the $8^{\text {th }}$ century and the first third of the $9^{\text {th }}$ century. These indirectly confirm the assumption of inhabitants of privileged rank at the hillfort in the Great Moravian period (Kouřil 2007).

\section{Conclusion}

Genetic comparison of the dog bone from ChotěbuzPodobora hillfort with genetic material from a modern greyhound has shown that the bone found most probably belonged to this breed.

Since ancient times the greyhound has been an exceptional animal which was only ever owned by nobles and rulers. The presence of a greyhound at the hillfort supports the theory that at the turn of the $8^{\text {th }}$ and $9^{\text {th }}$ centuries $\mathrm{AD}$ the fort was occupied by elite forces under the command of a local leader, who guarded the entrance to the Moravian Gate. It also implies that trade or political links existed with the region which is now Poland.

\section{Acknowledgments}

We thank Mgr. Radka Simonová Ph.D. for familiarization. We also wish to thank Mgr. Jiř́ Vidlák and Mgr. Jitka Panušková for their careful review of this manuscript. The greyhound rescue group in the Czech Republic is also thanked, especially and her dog for the gift of samples for comparative purposes. This research was carried out at PČR Ǩ̌P Jmk OKTE Brnoand GeneProof a.s. Brno. We gratefully acknowledge grant no. AVOZ80010507 from the Institute of Archaeology, Academy of Science of the Czech Republic.

\section{References}

Baron, H., Hummel, S., Herrmann, B. (1996): Mycobacterium tuberculosis complex DNA in ancient human bones. Journal of Archeological Sciences, 23: 667-671. http://dx.doi.org/10.1006/jasc. 1996.0063

Bazin, G. A. (1959): History of Art from Prehistoric Times to the Present. - Crown Publishers, New York, 250 pp.

Branigan, C. A. (2004): The Reign of the Greyhound. A popular History of the Oldest Family of Dogs. - Howell Book House, New Jersey, 256 pp.

Brentjes, B. (1969): African Rock Art. - J. M. Dent and Sons, London, 104 pp.

Buben, M. (2003): Encyklopedie heraldiky [Encyclopedia of Heraldry]. - Libri, Praha, 505 pp. (in Czech)

Císařovský, M. (2008): Pes [Dog]. - Altercan, Praha, 902 pp. (in Czech)

Gundry, R. L., Allard, M. W., Moretti, T. R., Honeycutt, R. L., Wilson, M. R., Monson, K. L., Foran, D. R. (2007): Mitochondrial DNA Analysis of the Domestic Dog: Control Region Variation Within and Among Breeds. Journal of Forensic Sciences, 52: 562-572. http://dx.doi.org/10.1111/j.1556-4029.2007.00425.x 
Harcourt, R. A. (1974): The Dog in Prehistoric and Early Historic Britain. - Journal of Archaeological Science, 1: 151-175. http://dx.doi.org/10.1016/0305-4403(74)90040-5

Herrmann, B., Hummel, S. (eds) (1994): Ancient DNA. Springer-Verlag, New York, 284 pp. http://dx.doi.org/10.1007/978-1-4612-4318-2

Horai, S., Hayasaka, K., Kondo, R., Tsugane, K., Takahata, N. (1995): Recent African origin of modern humans revealed by complete sequences of hominoid mitochondrial DNAs. - Proceedings of the National Academy of Sciences of the United States of America, 92: 532-536. http://dx.doi.org/10.1073/pnas.92.2.532

Hummel, S. (2003): Ancient DNA Typing. Methods, Strategies and Applications. - Springer, Berlin, New York, $298 \mathrm{pp}$. http://dx.doi.org/10.1007/978-3-662-05050-7

Kim K. S, Lee S. E., Jeong H. W., Ha J. H. (1998). The complete nucleotide sequence of the domestic dog (Canis familiaris) mitochondrial genome. - Molecular Phylogenetics and Evolution 10(2): 210-220. http://dx.doi.org/10.1006/mpev.1998.0513

Kholová, H. (1987): Historie psího rodu [History of dog's dynasty]. - Práce, Praha, 326 pp. (in Czech)

Kouřil, P. (1994): Slovanské osídlení Českého Slezska [Slavic Settlement in Czech Silesian]. - Archeologický ústav AV ČR and Muzeum Těšínska, Brno, Český Těšín, 220 pp. (in Czech)

Kouřil, P. (1997): Severní předpolí Moravské brány a zásah velkomoravský [The north Moravian Gate and the intervention of the Great Moravian]. - In: Wachowski, K. (ed.), Śląsk i Czechy a kultura wielkomorawska, Uniwersytet Wrocławski, Wrocław, p. 65-75. (in Czech)

Kouřil, P. (2007): Jezdecká elita na hradisku v ChotěbuziPodoboře [Equestion elite riders from Chotěbuz-Podobora hillfort]. - Těšínsko, 50: 5-8. (in Czech)

Morris, D. (2001): Dogs: The Ultimate Dictionary of over 1000 Dog Breeds. - Ebury Press, London, 752 pp.

Mysliveček, M. (1993): Erbovník. Aneb kniha o znacích a osudech rodů žijících v Čechách a na Moravě podle starých pramenů a dávných a ne vždy věrných svědectví [Coat of arms. Or a book about the characters and fates of families living in Bohemia and Moravia by the ancient sources and ancient and not always faithful witness]. Horizont, Praha, 159 pp. (in Czech)

Nývltová Fišáková, M. (2007): Nález chrta na hradisku Chotěbuz-Podobora [Greyhound find from ChotěbuzPodobora hillfort]. - Těšínsko, 50: 29-31. (in Czech)

Nývltová Fišáková, M. (2010): Local elite from ChotěbuzPodobora from zooarcheological and archeological point of view. - Animals as Material Culture in the Middle Ages. Bestial Mirrors VIAS (Vienna Institute of Archaeological Science), 2010(3): 31-38.

Reimer, P. J., Baillie, M. G. L., Bard, E., Bayliss, A., Beck, J. W., Bertrand, C., Blackwell, P. G., Buck, C. E., Burr, G., Cutler, K. B., Damon, P. E., Edwards, R. L., Fairbanks, R. G., Friedrich, M., Guilderson, T. P., Hughen, K. A., Kromer, B., McCormac, F. G., Manning, S., Ramsey, B. C., Reimer, R. W., Remmele, S., Southon, J. R., Stuiver, M., Talamo, S., Taylor, F. W., van der Plicht, J., Weyhenmeyer, C.E. (2013): IntCal13 and marine 13 radiocarbon age calibration curves 0-50,000 years Cal BP. - Radiocarbon, 55: 1869-1887. http://dx.doi.org/10.2458/azu_js_rc.55.16947

Stuchlý, I., Císařovský, M. (1992): Chrti [Greyhounds]. Canis, Praha, 143 pp. (in Czech)

Sullivan, M. (1999): The Ultimate Greyhound. - Interpret Publishing, London, $250 \mathrm{pp}$.

Vila, C., Savolainen, P., Maldonado, J. E., Amorim, I. R., Rice, J. E., Honeycutt, R. L., Crandall, K. A., Lundeberg, J., Wayne, R. K. (1997): Multiple and Ancient Origins of the Domestic Dog. - Science, 276: 1687-1689. http://dx.doi.org/10.1126/science.276.5319.1687

Walsh, P. S., Metzger, D. A., Higuchi, R. (1991): Chelex 100 as a medium for simple extraction of DNA for PCR-based typing from forensic material. - BioTechniques, 10: 506-513.

Webb, K. M., Allard, M. W. (2009): Identification of forensically informative SNPs in the domestic dog mitochondrial control region. - Journal of Forensic Science, 54: 289-304. http://dx.doi.org/10.1111/j.1556-4029.2008.00953.x

Yang, H., Goldberg, E. M., Shoshani, J. (1997): Prosboscidean DNA from museum and fossil specimens: an assessment of ancient DNA extraction and amplification techniques. - Biochemical Genetics, 35: 165-179. http://dx.doi.org/10.1023/A:1021902125382 